\title{
ON THE CALCULATION OF QUANTUM MECHANICAL GROUND STATES FROM CLASSICAL GEODESIC MOTION ON CERTAIN SPACES OF CONSTANT NEGATIVE CURVATURE
}

\author{
R. TOMASCHITZ \\ Sechsschimmelg. 1/21-22, A-1090 Vienna, Austria and KFA Jülich (IFF), D-5170 Jülich, FRG \\ Received 20 March 1988 \\ Accepted 23 June 1988 \\ Communicated by V.I. Arnol'd
}

\begin{abstract}
We consider geodesic motion on three-dimensional Riemannian manifolds of constant negative curvature, topologically equivalent to $S \times] 0,1[, S$ a compact surface of genus two. To those trajectories which are bounded and recurrent in both directions of the time evolution $t \rightarrow+\infty, t \rightarrow-\infty$ a fractal limit set is associated whose Hausdorff dimension is intimately connected with the quantum mechanical energy ground state, determined by the Schrödinger operator on the manifold.

We give a rather detailed and pictorial description of the hyperbolic spaces we have in mind, discuss various aspects of classical and quantum mechanical motion on them as far as they are needed to establish the connection between energy ground state and Hausdorff dimension and give finally some examples of ground state calculations in terms of Hausdorff dimensions of limit sets of classical trajectories.
\end{abstract}

\section{Introduction}

Geodesic motion on manifolds of constant negative curvature has often provided - due to the fact that such manifolds sometimes admit analytically very tractable normal forms though the trajectories behave very erratically - interesting examples for various dynamical concepts like ergodicity, entropy, mixing, Bernoulli property etc. (cf. e.g. Anosov [4], Pesin [37]).

In 1956 Selberg [40] and Huber [23] announced a deep connection between the eigenvalues of the Laplace-Beltrami operator (which is the Schrödinger operator as far as quantized geodesic motion is concerned) and the homotopy classes of geodesic trajectories on such manifolds, and they derived for this connection a relation which is nowadays called Selberg's trace formula (cf. e.g. McKean [32]).

This discovery initiated amongst many other things a thorough study of the spectral properties of the Laplace-Beltrami operator, mainly on Riemann surfaces (cf. e.g. [39, 15, 33, 22, 13]) but also on three-dimensional hyperbolic manifolds (cf. [16]). As an offspring of these investigations Patterson [34] derived an extremely interesting relation between the lowest eigenvalue of the Laplace-Beltrami operator and the Hausdorff dimension of a fractal set that emerges at infinity of hyperbolic space as the set of initial and end points of trajectories that are bounded and recurrent in both time directions $t \rightarrow+\infty, t \rightarrow-\infty$. This connection is exact, no kind of semiclassical approximation is involved.

While Selberg's trace in its present form (cf. e.g. [46, 22]) turned out to be numerically not accessible (even in two dimensions cf. [19,20,5]), the above-mentioned relation is, and will be used here to determine on some three-dimensional hyperbolic manifolds (i.e. Riemannian manifolds of constant sectional Gauss- 
ian curvature $-1 / R^{2}, R$ being the curvature radius of hyperbolic space) the energy ground state $E_{0}$ via

$$
E_{0}=\frac{\hbar^{2}}{R^{2} m} \delta_{\mathrm{H}}\left(1-\frac{\delta_{\mathrm{H}}}{2}\right)
$$

where $\hbar$ denotes Planck's quantity, $m$ the mass of the geodesically moving particle and $\delta_{\mathrm{H}}$ the Hausdorff dimension of the limit set.

The paper is written up as follows: In section 1 we give an elementary geometric description of the hyperbolic manifolds we will deal with (see also section 8), derive their normal forms in three-dimensional hyperbolic space, discuss various aspects of classical geodesic motion on them and explain the origin of the fractal set, whose Hausdorff dimension is involved in (0.1).

To derive formula (0.1) in section 4 we need some facts about the spectral theory of the Schrödinger operator, which we present in sections 2 (resolvent) and 3 (ground state eigenfunction). In sections 5 and 6 we provide practical construction techniques for hyperbolic manifolds and in section 7 a method to localize the limit set and to calculate its Hausdorff dimension.

In the final section 8 we present various examples of hyperbolic manifolds and calculate ground state energies of the quantized geodesic motion on them via Hausdorff dimensions of limit sets of classical trajectories.

\section{Hyperbolic manifolds, classical geodesic motion on them and the emergence of a fractal set}

The space in which our manifolds are modelled and from which their metric structure is inherited is Poincaré's upper half-space version of hyperbolic geometry: $H^{3}:=\left\{\left(y_{1}, y_{2}, y_{3}\right) \in \mathbb{R}^{3}, y_{3}>0\right\}$, endowed with a Riemannian metric of constant negative curvature $-1 / R^{2}$ (cf. e.g. $[3,8]$ ),

$$
\mathrm{d} s^{2}=\frac{R^{2}\left(\mathrm{~d} y_{1}^{2}+\mathrm{d} y_{2}^{2}+\mathrm{d} y_{3}^{2}\right)}{y_{3}^{2}} .
$$

Indeed, $H^{3}$ is isometric to the standard model of three-dimensional hyperbolic geometry, namely to the upper sheet $Q^{3}$ of a two-sheeted hyperboloid in $\mathbb{R}^{4}$,

$$
x_{0}^{2}-x^{2}=R^{2}, \quad x_{0}>0, \quad x:=\left(x_{1}, x_{3}, x_{3}\right),
$$

with the pseudo-Euclidean metric of $\mathbb{R}^{4}$,

$$
\mathrm{d} s^{2}=\mathrm{d} x^{2}-\mathrm{d} x_{0}^{2}
$$

restricted to it. For explicit formulae for the isometry $Q^{3} \leftrightarrow H^{3}$ see [8] or section 2 . The orientation-preserving invariance group of the metric (1.3) on $Q^{3}$ is the restricted orthochronous Lorentz group $\mathrm{SO}^{+}(3,1)$, acting on $Q^{3}$ by the standard matrix representation, and this group is known to be isomorphic to $\operatorname{SL}(2, \mathbb{C}) /\{ \pm$ id $\}$, the group of two by two complex matrices with determinant 1 ; the quotient simply means that two matrices differing by an overall sign are identified.

It is evident that $\operatorname{SL}(2, \mathbb{C}) /\{ \pm \mathrm{id}\}$ is isomorphic to the group of complex Möbius transformations in the extended complex plane $\mathbb{C} \cup\{\infty\}$ : To every matrix $A:= \pm\left(\begin{array}{ll}a & b \\ c & d\end{array}\right)$ in $\operatorname{SL}(2, \mathbb{C}) /\{ \pm$ id $\}$ we associate a 
Möbius transformation via

$$
z \rightarrow A z=\frac{a z+b}{c z+d}
$$

Every Möbius transformation can be realized by a successive performance of four (or fewer) inversions in circles or straight lines (e.g. [17]). An inversion in a circle in $\mathbb{R}^{2}$ or an inversion in a sphere in $\mathbb{R}^{3}$ with center $\boldsymbol{M}$ and radius $r$ is defined as

$$
x \rightarrow \zeta(x)=M+\frac{r^{2}}{|x-M|^{2}}(x-M)
$$

Observe that

$$
(\boldsymbol{x}-\boldsymbol{M})(\zeta(\boldsymbol{x})-\boldsymbol{M})=r^{2}
$$

In the case of inversion in a straight line in $\mathbb{R}^{2}$ or a plane in $\mathbb{R}^{3} \zeta(x)$ denotes the mirror image of $\boldsymbol{x}$ with respect to the straight line or the plane.

The complex plane is just the boundary of the hyperbolic half-space $H^{3}$, and we can lift the action (1.4) of $\operatorname{SL}(2, \mathbb{C}) /\{ \pm \mathrm{id}\}$ on $\mathbf{C}$ to $H^{3}$ as follows (Poincaré): We choose four or fewer circles or straight lines representing a given Möbius transformation via a succession of inversions, replace them by hemispheres in $H^{3} \cup \mathrm{C}$ with the same centers and radii or by half-planes orthogonal to $\mathrm{C}$, and define the lifted (extended) Möbius transformation in $H^{3} \cup \mathrm{C} \cup\{\infty\}$ as a succession of inversions in these hemispheres or half-planes. We list also the analytic expression for these lifted transformations (cf. [8]; we use complex notation, $\left.z:=y_{1}+\mathrm{i} y_{2}, \bar{z}:=y_{1}-\mathrm{i} y_{2}\right)$ :

$$
\begin{aligned}
& \gamma: H^{3} \rightarrow H^{3}, \\
& \gamma:\left(z, y_{3}\right) \rightarrow\left(\frac{(a z+b)(\overline{c z+d})+a \bar{c} y_{3}^{2}}{|c z+d|^{2}+|c|^{2} y_{3}^{2}}, \frac{y_{3}}{|c z+d|^{2}+|c|^{2} y_{3}^{2}}\right) .
\end{aligned}
$$

It is clear from both the definition and the expression (1.7) that the restriction of the lifted transformation to $\mathbb{C} \cup\{\infty\}\left(y_{3}=0\right)$ is still given by (1.4).

Moreover, the lifted transformations map $H^{3}$ bijectively onto itself and preserve the Poincaré metric (1.1). The standard matrix representation $\mathrm{SO}^{+}(3,1)$ on $Q^{3}$ is equivalent to the representation (1.7) of $\operatorname{SL}(2, \mathbb{C}) /\{ \pm \mathrm{id}\}$, acting via extended Möbius transformations on $H^{3}$. We refer to $\operatorname{SL}(2, \mathbb{C}) /\{ \pm \mathrm{id}\}$ as the orientation-preserving invariance group of three-dimensional hyperbolic space.

The extended Möbius transformations in $H^{3}$ inherit some important properties of the two-dimensional ones in the complex plane. Two-dimensional transformations (1.4) map circles and straight lines onto circles and straight lines (cf. e.g. [17]), and the extended transformations map hemispheres in $H^{3}$ with centers in the complex plane and half-planes orthogonal to the complex plane again onto hemispheres centered in $\mathbf{C}$ and half-planes orthogonal to $\mathbf{C}$. In order to imagine the action of the lifted transformation in $H^{3} \cup \mathrm{C} \cup\{\infty\}$ it is therefore always useful to look at the base circles of the hemispheres resting on the complex plane, which are transformed by (1.4). Just as the two-dimensional transformations (1.4) are bijective, conformal (i.e. angle preserving) maps of $\mathbb{C} \cup\{\infty\}$ onto itself, the extended transformations in $H^{3}$ also enjoy these properties. 
We need also some facts about geodesic motion in $H^{3}$. As a straightforward generalization of free motion in Euclidean space, the Lagrange function for geodesic motion in a curved Riemannian space with line element

$$
\mathrm{d} s^{2}=\sum_{i, j=1}^{3} g_{i j}(x) \mathrm{d} x_{i} \mathrm{~d} x_{j}
$$

is given by

$$
L=\frac{1}{2} m \sum_{i, j=1}^{3} g_{i j}(x) \dot{x}_{i} \dot{x}_{j} \equiv E .
$$

(The overdots denote time derivatives.) The identity means that $L$ is a constant of motion, for the $\left(g_{i j}\right)$ are time independent.

In the case of the line element (1.1) we have

$$
L=\frac{1}{2} m \frac{R^{2}}{y_{3}^{2}}\left(\dot{y}_{1}^{2}+\dot{y}_{2}^{2}+\dot{y}_{3}^{2}\right) \equiv E .
$$

It is clear from this expression that the component of the initial direction in the $\left(y_{1}, y_{2}\right)$-plane is left unchanged by the motion; the particle remains always in a half-plane orthogonal to $\mathbb{C}$ determined by the initial direction. Denoting by $x$ and $y$ Cartesian coordinates in this half-plane we have

$$
L=\frac{1}{2} m \frac{R^{2}}{y^{2}}\left(\dot{x}^{2}+\dot{y}^{2}\right) \equiv E .
$$

As a second constant of motion we have

$$
\frac{\partial L}{\partial \dot{x}}=m \frac{R^{2}}{y^{2}} \dot{x} \equiv p
$$

Combining (1.10) and (1.11) we get

$$
E=\frac{1}{2} \frac{1}{R^{2}} \frac{p^{2}}{m} y^{2}\left(1+\left(\frac{\mathrm{d} y}{\mathrm{~d} x}\right)^{2}\right)
$$

Solving this equation we see that the geodesics are arcs of semicircles orthogonal to the $\left(y_{1}, y_{2}\right)$-plane,

$$
\left(x-x_{0}\right)^{2}+y^{2}=\frac{2 E R^{2} m}{p^{2}} .
$$

In the case that $p=0$ we have instead of (1.13) $x=x_{0}$, i.e. the trajectories are straight lines perpendicular onto the $\left(y_{1}, y_{2}\right)$-plane. To get the time dependence of the motion we have also from (1.10) and (1.11)

$$
\left(\frac{\mathrm{d} y}{\mathrm{~d} t}\right)^{2}=\frac{2 E y^{2}}{m R^{2}}-p^{2} \frac{y^{4}}{m^{2} R^{4}}
$$


or

$$
y(t)=\frac{R \sqrt{2 m E}}{p} \frac{1}{\cosh \left(\frac{\sqrt{2 E / m}}{R}\left(t-t_{0}\right)\right)},
$$

with

$$
y_{0}:=y\left(t_{0}\right)=\frac{R \sqrt{2 m E}}{p}
$$

if $p \neq 0$ (cf. also (1.13)).

In the case that $p=0$ we have instead of (1.15)

$$
y(t)=y_{0} \exp \left(\frac{\sqrt{2 E / m}}{R}\left(t-t_{0}\right)\right),
$$

where $y_{0}$ can be chosen freely.

From (1.13), (1.15) and (1.16) we also get

$$
\sinh ^{2}\left(\frac{1}{2} \frac{\sqrt{2 E / m}}{R}\left(t-t_{0}\right)\right)=\frac{\left(x-x_{0}\right)^{2}+\left(y-y_{0}\right)^{2}}{4 y y_{0}},
$$

which is $p$-independent and remains also true for $p=0$. From (1.8) and (1.10) it is evident that $\sqrt{2 E / m}\left(t-t_{0}\right)$ is just the hyperbolic distance between $\left(x_{0}, y_{0}\right)$ and $(x, y)$ in $H^{3}(x, y$ are coordinates in a half-plane in $H^{3}$ orthogonal to C).

The construction principle of three-dimensional hyperbolic manifolds (hyperbolic means here that they can be endowed with a Riemannian metric of constant negative curvature) in hyperbolic space $H^{3}$ is best explained by a two-dimensional Euclidean example:

One can represent a Riemann surface of genus one (a torus) as a square $\mathscr{F}$ in $\mathbb{R}^{2}$ with vertices $(0,0)-(1,0)-(1,1)-(0,1)$, the sides $(0,0)-(1,0)$ and $(1,0)-(1,1)$ being identified by the Euclidean translation $(x, y) \rightarrow(x, y)+(0,1)$ and the other two sides by the translation $(x, y) \rightarrow(x, y)+(1,0)$. This torus inherits a Riemannian metric of zero curvature from $\mathbb{R}^{2}$ by restricting the Euclidean metric $\mathrm{d} s^{2}=\mathrm{d} x^{2}+\mathrm{d} y^{2}$ of $\mathbb{R}^{2}$ to the square. That this metric fits also smoothly on the sides which are identified by the above two transformations is due to the fact that $\mathrm{d} s^{2}$ is invariant under these transformations and that the square is a fundamental domain for the subgroup $\Gamma$ of Euclidean translations generated by these two translations. Fundamental domain means here that if we apply all elements of $\Gamma$ to the square we get a tesselation of $\mathbb{R}^{2}$ with squares which do not overlap and cover all of $\mathbb{R}^{2}$.

Every geodesic on the torus $\mathscr{F}_{\mathrm{M}}$, i.e. the unit square $\mathscr{F}$ with identified sides can be obtained by choosing suitably a geodesic $s$ in $\mathbb{R}^{2}$ and projecting it into $\mathscr{F}$. The projection map $\pi$ is defined so, that if a square $\gamma(\mathscr{F}), \gamma \in \Gamma$, in the tesselation of $\mathbb{R}^{2}$ contains a piece of the geodesic in $\mathbb{R}^{2}$, this piece is mapped into $\mathscr{F}$ by $\gamma^{-1}$. (Technically speaking, $\pi$ is the natural projection map of the universal covering space $\mathbb{R}^{2}$ of $\mathscr{F}_{\mathrm{M}}$ onto $\mathscr{F}_{\mathrm{M}} \cong \mathbb{R}^{2} / \Gamma$.) So, for example, if we have a straight line $s$ in $\mathbb{R}^{2}$ that encloses the angle $\varphi$ with the side $(0,0)-(1,0)$ of $\mathscr{F}$ and $\tan \varphi$ is a rational number then $\pi(s)$ is a closed loop on $\mathscr{F}_{\mathrm{M}}$. If $\tan \varphi$ is irrational the image $\pi(s)$ is dense in $\mathscr{F}$, the trajectory comes arbitrarily close to every point of the torus. 


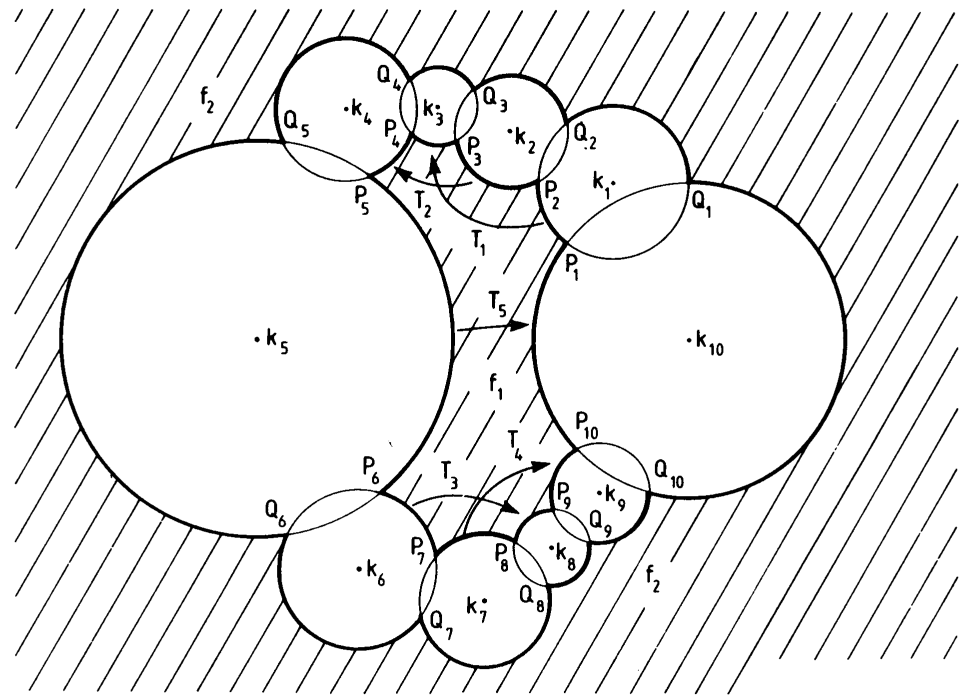

Fig. 1. Identification pattern for the base circles of ten intersecting hemispheres in the plane at infinity of $H^{3}$, giving rise to two compact surfaces of genus two (hatched), the boundary components of our hyperbolic manifolds; see sections 1, 5,8 .

Quite analogously to this two-dimensional example we model now manifolds $\mathscr{F}_{\mathrm{M}}$ in $H^{3}$ by constructing a domain $\mathscr{F}$ bordered by geodesic planes (domains on hemispheres centered in the complex plane) and by identifying these planes via lifted Möbius transformations, so that we get topologically the desired manifold $\mathscr{F}_{\mathrm{M}}$ (which is in our case homeomorphic to $\left.S \times\right] 0,1[, S$ a compact surface of genus two, i.e. a sphere with two handles). $\mathscr{F}$ must be a fundamental domain for the discrete group $\Gamma$ of lifted Möbius transformations that is generated by the identifying transformations, which means $\Gamma$ applied to $\mathscr{F}$ tesselates $H^{3}\left(\Gamma(\mathscr{F})\right.$ must completely cover $H^{3}$ without overlaps). If this is the case, the invariance of the Poincaré metric (1.1) under $\Gamma$ guarantees that its restriction to $\mathscr{F}$ defines on the manifold $\mathscr{F}_{\mathrm{M}}$ a smooth metric of constant sectional Gaussian curvature $-1 / R^{2}$ (cf. [31]).

The identification pattern for the manifold $S \times] 0,1[, S$ a compact surface of genus two, is presented in fig. 1: We have drawn the base circles of ten hemispheres centered in the complex plane, the boundary of $H^{3}$. These hemispheres intersect just in the prescribed way. The domain $\mathscr{F}$ consists of the region exterior to all ten hemispheres in $\mathrm{H}^{3}$ together with its bordering sides (geodesic planes) on the hemispheres. The manifold $\left.\mathscr{F}_{M} \cong S \times\right] 0,1\left[\left(\cong:\right.\right.$ homeomorphic) is just $\mathscr{F}$ with the side identification by the $T_{i}, i=1, \ldots, 5$. Every $T_{i}$ (a lifted Möbius transformation) identifies two boundary planes by mapping the corresponding two hemispheres onto one another, so that (with the notation of fig. 1): $T_{1}\left(P_{1}\right)=P_{4}, T_{1}\left(P_{2}\right)=P_{3}$, $T_{2}\left(P_{2}\right)=P_{5}, T_{2}\left(P_{3}\right)=P_{4}, T_{3}\left(P_{6}\right)=P_{9}, T_{3}\left(P_{7}\right)=P_{8}, T_{4}\left(P_{7}\right)=P_{10}, T_{4}\left(P_{8}\right)=P_{9}, T_{5}\left(P_{5}\right)=P_{1}, T_{5}\left(P_{6}\right)=P_{10}$, and the same relations with $P_{i}$ replaced by $Q_{i}$.

Let us for the moment attach the two bordering surfaces to $S \times] 0,1[$, that makes it a compact manifold $S \times[0,1]$. In $H^{3} \cup \mathcal{C} \cup\{\infty\}$ these two surfaces are presented (see fig. 1) by the interior $f_{1}$ and $f_{2}$ of two closed polygons with vertices $P_{i}, i=1, \ldots, 10$ and $Q_{i}, i=1, \ldots, 10$ (the second encloses the point at infinity), their circular arcs identified by the $T_{i}$. It is easy to imagine that this side identification gives rise to two compact surfaces of genus two. The other fibers of $S \times[0,1]$ can also easily be located in $\mathscr{F}$. For more pictorial details of the homeomorphism $\left.\mathscr{F}_{\mathrm{M}} \cong S \times\right] 0,1[$ see Marden [29] and section 8 .

Just as in our two-dimensional torus example we can realize geodesic motion on the hyperbolic manifold $\mathscr{F}_{\mathrm{M}}$ by projecting geodesics in $H^{3}$ (semicircles orthogonal to the complex plane) into $\mathscr{F}_{\mathrm{M}}$. The projection 
pp. $48-87$ in attached files 


\section{Acknowledgements}

I thank N.L. Balazs, M.C. Gutzwiller, S.J. Patterson and J. Villain for special help, and B.N. Apanasov, J. Elstrodt and T. Pignataro for useful correspondence on the preprint version of this work. An exciting stay at the KFA Jülich, where parts of this work were carried out, is also acknowledged.

\section{Note added in proof}

The Hausdorff dimensions quoted in the final section have been calculated by determining the slope of $\lambda \rightarrow \log \hat{N}(\lambda)$ (cf. tables I-V) via a slightly weighted Gaussian method of least squares. The weights 1 , $\log \lambda, \log ^{2} \lambda$ have been tried, the results remain unaffected by them to the accuracy given in section 8 .

I would like to thank S.J. Patterson for suggesting this method. I also add the following citations: There is the impressive thesis [55] of Thea Pignataro, dealing with conformal densities (measures) on limit sets, spectral theory, quantization of geodesic flows, and all that. Here and in [56] it is shown how to express eigenfunctions as weighted integrals over classical orbits.

There are two competent reviews [53, 57], more lucid information on fundamental domains and tesselations [50, 51] and hyperbolic spaces [59-61], additional work on spectral theory and Eisenstein series $[48,52,54,58]$, and the Gelfand school [49] with the $\operatorname{SL}(2, \mathbb{C})$ trace formula.

\section{References}

[1] W. Abikoff, Topics in the Real Analytic Theory of Teichmüller Space, Lecture Notes in Mathematics, vol. 820 (Springer, Berlin, 1980).

[2] L.V. Ahlfors, Lectures on Quasiconformal Mappings (Van Nostrand, New York, 1966).

[3] L.V. Ahlfors, Möbius Transformations in Several Dimensions, Lecture Notes (Univ. Minnesota, 1981).

[4] D.U. Anosov, Proc. Steklov Inst. Math. 90 (1967) 1; (AMS Transl., Providence, R.I., 1969).

[5] N.L. Balazs and A. Voros, Phys. Rep. 143 (1986) 109.

[6] A.F. Beardon, Am. J. Math. 88 (1966) 722.

[7] A.F. Beardon and B. Maskit, Acta Math. 132 (1974) 1.

[8] A.E. Beardon, The Geometry of Discrete Groups, Graduate Texts in Mathematics, vol. 91 (Springer, Berlin, 1983).

[9] L. Bers, Ann. Math. 91 (1970) 570.

[10] L. Bers, Bull. London Math. Soc. 4 (1972) 257.

[11] L. Bers, Bull. Am. Math. Soc. (New Series) 5 (1981) 131.

[12] L. Carleson, Selected Problems on Exceptional Sets, Van Nostrand Math. Studies, vol. 14 (1967).

[13] Y. Colin de Verdière, Ann. Inst. Fourier 32 (1982) 275; 33 (1983) 87.

[14] C.J. Earle, in: Discrete Groups and Automorphic Functions, W.J. Harvey, ed. (Academic Press, London, 1977).

[15] J. Elstrodt, Math. Ann. 203 (1973) 295; 208 (1974) 99.

[16] J. Elstrodt, F. Grunewald and J. Mennicke, Russ. Math. Surv. 38 (1983) 137.

[17] L. Ford, Automorphic Functions (Chelsea, Bronx, 1951).

[18] L. Greenberg, in: Discrete Groups and Automorphic Functions, W.J. Harvey, ed. (Academic Press, London, 1977).

[19] M.C. Gutzwiller, Phys. Rev. Lett. 45 (1980) 150.

[20] M.C. Gutzwiller, Physica D5 (1982) 183.

[21] M.C. Gutzwiller, Physica D7 (1983) 341.

[22] D.A. Hejhal, The Selberg Trace Formula for PSL(2,R), vol. 1,2, Lecture Notes in Mathematics Nr. 548, Nr. 1001 (Springer, Berlin, 1976 and 1983).

[23] H. Huber, Comm. Math. Helv. 30 (1956) 20. 
[24] S.L. Krushkal, B.N. Apanasov and N.A. Grusevskii, Kleinian Groups and Uniformization in Examples and Problems, Translations of Math. Monographs, vol. 62 (AMS, 1986).

[25] P.D. Lax and R.S. Phillips, J. Funct. Anal. 46 (1982) 280.

[26] J. Lehner, Discontinuous Groups and Automorphic Functions (AMS, Providence, R.I., 1964).

[27] W. Magnus, F. Oberhettinger and R.P. Soni, Formulas and Theorems for the Special Functions of Mathematical Physics, Grundlehren, vol. 52 (Springer, Berlin, 1966).

[28] A. Marden, Ann. Math. 99 (1974) 383.

[29] A. Marden, in: Discrete Groups and Automorphic Functions, W.J. Harvey, ed. (Academic Press, London, 1977).

[30] A. Marden, Bull. Am. Math. Soc. (New Series) 3 (1980) 1001.

[31] B. Maskit, Adv. Math. 7 (1971) 219.

[32] H.P. McKean, Comm. Pure Appl. Math. 35 (1972) 225.

[33] S.J. Patterson, Compositio Math. 31 (1975) 83; 32 (1976) 71; 33 (1977) 227.

[34] S.J. Patterson, Acta Math. 136 (1976) 241.

[35] S.J. Patterson, Math. Proc. Camb. Phil. Soc. 81 (1977) 59.

[36] D. Pedoe, A Course of Geometry (Cambridge Univ. Press, 1970).

[37] Ya.B. Pesin, Russ. Math. Surv. 32 (1977) 55.

[38] R.S. Phillips and P. Sarnak, Acta Math. 155 (1985) 173.

[39] W. Roelcke, Math. Ann. 167 (1966) 292; 168 (1967) 261.

[40] A. Selberg, J. Ind. Math. Soc. 20 (1956) 47.

[41] D. Sullivan, Publ. Math. I.H.E.S. 50 (1979) 171.

[42] D. Sullivan, Bull. Am. Math. Soc. (New Series) 6 (1982) 57.

[43] D. Sullivan, Acta Math. 153 (1984) 259.

[44] W. Thurston, The Geometry and Topology of 3-Manifolds, Lecture Notes (Princeton Univ., 1978).

[45] W. Thurston, Bull. Am. Math. Soc. (New Series) 6 (1982) 357.

[46] A.B. Venkov, Russ. Math. Surv. 34 (1979) 79.

[47] D. Wright, Oklahoma State Univ., in preparation.

[48] L.D. Faddeev, Trans. Moscow Math. Soc. 17 (1967) 357.

[49] I.M. Gelfand, M.I. Graev, I.I. Pyatetskii-Shapiro, Representation Theory and Automorphic Functions (Saunders, Philadelphia, 1969).

[50] L. Keen, Ann. of Math. 84 (1966) 404.

[51] J.W. Magnus, Non-Euclidean Tesselations and their Groups (Academic Press, New York, 1974).

[52] N. Mandouvalos, Eisenstein Series for Kleinian Groups, I.H.E.S.-preprint, 1984.

[53] S.J. Patterson, in Analytical and Geometrical Aspects of Hyperbolic Space, D.B.A. Epstein, ed., London Math. Soc. Lecture Notes 111 (Cambridge Univ. Press, London, 1987).

[54] S.J. Patterson, Mathematica Gottingensis, Heft 78, 1986.

[55] T. Pignataro, PhD-Thesis, Princeton, 1984.

[56] T. Pignataro and A.S. Wightman, in Group Theoretical Methods in Physics, Proc., W. Zachary, ed., (World Scientific, Singapore, 1984).

[57] D. Sullivan, in Aspects of Mathematics and its Applications, J.A. Barroso, ed., (Elsevier, Amsterdam, 1986).

[58] D. Hejhal, Boundary-Groups, Degenerating Riemann Surfaces and Spectral Theory, preprint, Univ. of Minnesota, 1987.

[59] B.N. Apanasov, The Geometry of Discrete Groups (Reidel, Dordrecht), to appear.

[60] D.B.A. Epstein and A. Marden, in: Analytical and Geometrical Aspects of Hyperbolic Space, London Math. Soc. Lecture Notes 111, D.B.A. Epstein, ed. (Cambridge Univ. Press, London, 1987).

[61] B.N. Apanasov, Ann. Global Anal. Geom. 6 (1988) 207. 